Swarthmore College

Works

12-1-1996

\title{
Spheromak Experiment Using Separate Guns For Formation And Sustainment
}

Michael R. Brown

Swarthmore College, doc@swarthmore.edu

A. Martin

Follow this and additional works at: https://works.swarthmore.edu/fac-physics

Part of the Physics Commons

Let us know how access to these works benefits you

\section{Recommended Citation}

Michael R. Brown and A. Martin. (1996). "Spheromak Experiment Using Separate Guns For Formation And Sustainment". Fusion Science And Technology. Volume 30, Issue 3 Pt. 1. 300-309.

https://works.swarthmore.edu/fac-physics/123

This work is brought to you for free by Swarthmore College Libraries' Works. It has been accepted for inclusion in Physics \& Astronomy Faculty Works by an authorized administrator of Works. For more information, please contact myworks@swarthmore.edu. 


\title{
SPHEROMAK EXPERIMENT USING SEPARATE GUNS FOR FORMATION AND SUSTAINMENT
}

EXPERIMENTAL DEVICES

KEYWORDS: spheromak, sustainment, current drive

\author{
MICHAEL R. BROWN Swarthmore College \\ Department of Physics and Astronomy, Swarthmore, Pennsylvania 19081
}

ADAM MARTIN University of Washington

Department of Physics and Astronomy, Seattle, Washington 98195

Received May 31, 1995

Accepted for Publication March 23, 1996

An experiment is described that incorporates the use of separate magnetized plasma guns for formation and sustainment of a spheromak. It is shown that energy coupling efficiency approaches unity if the gun and spheromak are of comparable size. A large gun should be able to operate at lower current and therefore lower voltage. In addition, it is expected that a gun matched to the size of the spheromak will cause less perturbation to the equilibrium. It is proposed to use a smaller gun for spheromak formation and a large, efficient gun for sustainment. The theoretical basis for the experiment is developed, and the details of the experiment are described. A prediction of the equilibrium magnetic flux surfaces using the EFIT code is presented.

\section{INTRODUCTION}

A spheromak is an example of a "force-free" magnetofluid configuration in which the internal forces (which are largely magnetic $\boldsymbol{J} \times \boldsymbol{B}$ forces) are in approximate balance. If $\boldsymbol{J} \times \boldsymbol{B} \cong 0$, then the magnetic fields in the force-free magnetofluid configuration obey the eigenvalue equation $\nabla \times \boldsymbol{B}=\lambda \boldsymbol{B}$, where $\lambda$ is a constant. Because of their relative simplicity, force-free states have been studied as magnetic confinement fusion configurations.'

A magnetofluid evolves into a force-free state through a turbulent process known as "relaxation." Turbulence, allied with small resistivity, allows the plasma rapid access (in a time that is short compared with the usual resistive diffusion time) to a minimum-energy force-free state. 'An important quantity in this relaxation process is the magnetic helicity of the configuration $K=\int \boldsymbol{A} \cdot \boldsymbol{B} d^{3} x$.
Helicity is a conserved quantity in ideal magnetohydrodynamics (MHD). It has the units of (magnetic flux) ${ }^{2}$ and is a measure of the degree to which pairs of closed flux tubes in a magnetofluid are linked. It can also be viewed as a volume-averaged measure of the "twistedness" of the magnetic field lines and is related to field aligned current. The remarkable property of magnetic helicity is that it is conserved even in the presence of turbulence (under conditions where magnetic energy is rapidly dissipated). This was suggested by Taylor ${ }^{2}$ and verified experimentally in magnetic fusion configurations such as spheromaks and reversed field pinches.

It can be shown ${ }^{1}$ that if the magnetic energy of a magnetofluid in a perfectly conducting vessel $W=$ $\int\left[B^{2} / 2 \mu_{0}\right] d^{3} x$ is minimized subject to the constraint that the magnetic helicity is fixed (using the technique of Lagrange multipliers), the resulting magnetic states satisfy the force-free condition $\nabla \times \boldsymbol{B}=\lambda \boldsymbol{B}$. The quantity $\lambda$ (the Lagrange multiplier in the calculation) is a constant eigenvalue with units of (length) ${ }^{-1}$. The value $\lambda$ is a measure of the ratio of current density to magnetic field $\left(\mu_{0} J / B\right)$ in the plasma as well as the ratio of energy to helicity $\left(2 \mu_{0} W / K\right)$.

Spheromaks can be sustained near the force-free state by continually injecting helicity (and energy) at a rate that balances helicity (and energy) dissipation. The efficiency of this process depends on the relative sizes of the spheromak and helicity injector. If spheromaks are to be considered as a viable alternative to tokamaks, it is important that efficient helicity injectors be developed for sustainment. We feel that the understanding of efficient helicity injection in spheromaks in the 1990s is analogous to the understanding of steady-state current drive for tokamaks in the 1970s. We propose to study the efficiency, impedance, and $\lambda$ of several types of magnetized plasma gun helicity sources on the Swarthmore Spheromak Experiment (SSX). In Sec. II, we briefly review the relevant spheromak work in other laboratories, 
and we develop the theoretical framework for gun efficiency in Sec. III. The existing facility is described in Sec. IV, and the proposed experiment is presented in Sec. V.

\section{PREVIOUS WORK}

Experimental work on spheromaks was begun in the early 1980s with the Compact Toroid Experiment (CTX) program at Los Alamos National Laboratory (LANL) (Ref. 3) and the S1 program at Princeton University, ${ }^{4}$ among others. The goal of this early work was to explore the spheromak as a magnetic confinement fusion configuration. Although spheromak formation proved to be relatively straightforward, researchers found that these plasmas were plagued by low temperatures, short confinement times, and instabilities (notably the tilt instability). In addition, the conversion of energy stored in capacitor banks to energy stored in the spheromak was found to be highly inefficient in early spheromak experiments.

By the late 1980s, the CTX group had produced stable $3 \mathrm{~T}$ spheromaks with electron temperatures in excess of $400 \mathrm{eV}$. (during decay) and decay times ${ }^{5}$ of several milliseconds. This success was due in part to the use of close-fitting, solid flux conservers and proper wall conditioning. ${ }^{6-9}$ The key to proper conditioning was the incorporation of titanium gettering of the flux conserver walls. ${ }^{10,11}$ In addition, the CTX group had perfected the slow-formation technique ${ }^{12}$ so that spheromaks could be sustained for times that were long compared with their resistive decay times.

The CTX group developed a magnetized, coaxial plasma gun to form and sustain spheromaks. The gun consists of an inner electrode that is magnetized by an external coil. Magnetic flux $\Phi_{\text {gun }}$ links the inner to the outer electrode. Gas is puffed into the annular gap, and high voltage is applied. The high voltage breaks down the gas, and current flows from the inner to the outer electrode generating toroidal magnetic flux, which encircles the inner electrode. If the $\boldsymbol{J} \times \boldsymbol{B}$ force is sufficient, then the magnetofluid and entrained toroidal flux distends the gun flux, and a spheromak is formed with $\Phi_{\text {gun }}$ becoming the poloidal flux. The voltage that appears between the inner and outer electrode is determined by the rate at which toroidal flux is ejected from the gun, ${ }^{13} V_{g u n}=d \Phi_{T o r} / d t$. During formation, helicity is injected at a rate $d K / d t=$ $2 V_{\text {gun }} \Phi_{\text {gun }}$. As the spheromak relaxes to the force-free state, some of the injected toroidal flux twists with respect to the magnetofluid and reconnects with the poloidal flux. By 1990, the impedance and efficiency of the coaxial plasma gun was well understood, and efficient guns were experimentally demonstrated. ${ }^{13}$

The principle of spheromak formation is analogous to the formation of a soap bubble. The analog of the surface tension of the soap film is the magnetic tension of the gun flux or what is known as the "stuffing flux" of the plasma gun. The analog of the air pressure that distends the soap film is the magnetic pressure due to the large discharge current of the plasma gun. If enough discharge current is supplied to the magnetized gun (usually on the order of $100 \mathrm{kA}$ ), then a spheromak (a kind of "magnetic bubble") is formed.

The Swarthmore Magnetofluids Laboratory will pursue techniques developed at the CTX laboratory. We will use the slow-formation technique and study the relaxation of the spheromak to the force-free state. The versatility of the device will allow us to try guns and flux conservers of different sizes so that we can compare the efficiency, impedance, and $\lambda$ of each configuration. To generate the hottest, cleanest spheromaks for magnetofluids experiments, we will use close-fitting, titaniumgettered copper flux conservers. Our spheromaks should have magnetic Reynolds numbers of several hundred for $T_{e}=10 \mathrm{eV}$. However, if we can increase $T_{e}$ to $50 \mathrm{eV}$, then we increase $R_{m}$ to several thousand.

\section{THEORETICAL FRAMEWORK}

\section{III.A. Gun Efficiency}

Recall that since the magnetic helicity $K=\int \boldsymbol{A} \cdot \boldsymbol{B} d^{3} x$ is related to the linked flux in the spheromak, it is also true that $K \sim I_{p}^{2}$. This suggests that helicity injection is tantamount to spheromak current drive. We have also noted that minimizing magnetic energy $W$ subject to the constraint of constant helicity $K$ results in the force-free condition $\nabla \times \boldsymbol{B}=\lambda \boldsymbol{B}$. Here

$$
\lambda=\frac{\boldsymbol{B} \cdot(\nabla \times \boldsymbol{B})}{\boldsymbol{B} \cdot \boldsymbol{B}}=\frac{\mu_{0} J_{\|}}{\boldsymbol{B}}
$$

is a measure of the inverse of the natural scale length of the system (either gun or spheromak). If we integrate the numerator and denominator of Eq. (1) over the surface area of the inner electrode of the gun, we have an effective $\lambda$ at the gun:

$$
\lambda_{\text {gun }} \simeq \frac{\mu_{0} I_{\text {gun }}}{\Phi_{\text {gun }}} .
$$

If we multiply the numerator and denominator of Eq. (1) by an effective parallel electric field and integrate over the volume of the spheromak discharge, we find an effective $\lambda$ for the spheromak:

$$
\lambda_{s p h} \simeq \frac{\mu_{0} \int J E_{\text {eff }} d^{3} x}{\int B E_{\text {eff }} d^{3} x} \simeq \frac{2 \mu_{0} \dot{W}}{\dot{K}} \simeq \frac{2 \mu_{0} W_{s p h}}{K_{s p h}},
$$

where in the last step we have integrated over time. This equation is also obtained as a result of the minimization process, where $\lambda_{s p h}$ is the Lagrange multiplier. It is clear that in any spheromak experiment, careful measurements 
of $\lambda_{s p h}$ and $\lambda_{g u n}$ (using the techniques outlined by Barnes et al. ${ }^{13}$ ) are important.

A simple equation governing helicity balance in the spheromak can be written as follows ${ }^{14,15}$ :

$$
\frac{\partial K_{s p h}}{\partial t}=-\frac{K_{s p h}}{\tau_{K}}+2 V_{g u n} \Phi_{g u n},
$$

where helicity is injected into the discharge at the rate $2 V_{\text {gun }} \Phi_{\text {gun }}$ and helicity is dissipated like $K_{s p h} / \tau_{K}$. If we replace $K_{s p h}$ with $W_{s p h}$ in the helicity balance equation using Eq. (3), we find an equation governing energy balance between the source (gun) and the spheromak:

$$
\frac{\partial W_{s p h}}{\partial t}=-\frac{W_{s p h}}{\tau_{K}}+\frac{\lambda_{s p h} V_{g u n} \Phi_{g u n}}{\mu_{0}}
$$

where we have assumed that $\partial \lambda_{s p h} / \partial t=0$ during steady state. If we replace $\Phi_{\text {gun }}$ with $I_{\text {gun }}$ in Eq. (5) using Eq. (2), we find

$$
\frac{\partial W_{s p h}}{\partial t}=-\frac{W_{s p h}}{\tau_{K}}+\frac{\lambda_{s p h}}{\lambda_{g u n}} V_{g u n} I_{g u n} .
$$

Notice that if the spheromak and the gun are the same size, then the $\lambda$ 's for each are the same. If $\lambda_{s p h}=\lambda_{\text {gun }}$, then in steady state, the gun power $V_{\text {gun }} I_{\text {gun }}$ simply balances magnetic energy loss $W_{s p h} / \tau_{K}$ in the spheromak. This is essentially ohmic current drive. We can identify an efficiency:

$$
\epsilon=\frac{\lambda_{s p h}}{\lambda_{g u n}} \sim \frac{r_{g u n}}{r_{s p h}}
$$

which approaches unity if the size of the gun and spheromak are comparable. Note that $\epsilon$ is a purely geometrical quantity that favors a plasma gun comparable to the size of the flux conserver. It is clear that during steadystate sustainment, we would like $\epsilon$ to approach unity; i.e., we would like to have the spheromak and the sustainment gun of comparable size.

\section{III.B. Gun Impedance}

Because the gun is an inductive load, the voltage that appears across the gun electrodes is not the applied capacitor voltage but is due to the ejection of toroidal flux:

$$
V_{\text {gun }}=\frac{\partial \Phi_{T o r}}{\partial t}=B_{\text {Tor }} \delta v_{\text {flow }},
$$

where $\delta$ is the interelectrode gap and $v_{\text {flow }}$ is the flow velocity of toroidal flux and plasma out of the gun. To compute the flow velocity, we note that in steady state, $v_{\text {flow }}$ is fixed, and the $J \times B$ force acting on the plasma is

$$
F=\frac{\partial(m v)}{\partial t}=\frac{\partial m}{\partial t} v_{\text {flow }}=\delta I_{\text {gun }} B_{\text {Tor }},
$$

where $m$ is the plasma mass. Because $B_{T o r}=\mu_{0} I_{\text {gun }} /$ $2 \pi r$, we note that the flow velocity contains two factors of $I_{\text {gun }}$, and so from Eq. (8), $V_{\text {gun }}$ is proportional to $I_{\text {gun }}^{3}$ :

$$
V_{\text {gun }}=\left(\frac{\mu_{0} \delta}{2 \pi r}\right)^{2} \frac{I_{g u n}^{3}}{\dot{m}} \text {. }
$$

This is essentially the same result obtained by Barnes et al. ${ }^{13}$ Measurements of $V_{\text {gun }}$ and $I_{\text {gun }}$ are straightforward. A direct measurement of the mass flow rate $\dot{m}=$ $\partial m / \partial t$ would help corroborate this model.

The normalized mass flow rate is sometimes referred to as the replacement factor or Morozov parameter $^{13}: \Xi=\left(I_{\text {gun }} / \dot{m}\right)(M / e)$. Here $\dot{m}$ is the rate mass that is ejected from the gun, and $M$ is the total mass of the plasma (so $\dot{m} / M$ is the analog of $I_{g u n} / e$ ). The value $\Xi$ is a measure of the number of times an electron is lost and needs to be replaced as a discharge proceeds. The value $\Xi$ is also referred to as the Hall parameter since it is the ratio of the Hall term $(J \times B / n e)$ to the inductive term $(\boldsymbol{v} \times \boldsymbol{B})$ in the generalized Ohm's law (where a suitable surface integral is performed). If $\Xi$ becomes appreciable, then ideal MHD becomes invalid. In CTX, it was determined that $\Xi \ll 1$, so the assumption of ideal MHD (co-moving ions and electrons) was a good one. However, it is interesting to note that for helicity injection current drive and steady-state sustainment of spheromaks, $\Xi \gg 1$ is desirable (i.e., it is better to inject current rather than particles). In tokamak jargon, efficient current drive schemes require a large $J / n$ (which is related to $\Xi$ ). The appropriate theory in this regime is Hall MHD rather than conventional MHD, suggesting that further theoretical investigations are in order. This will be particularly true if we are able to experimentally demonstrate a gun with $\epsilon \rightarrow 1$ and finite $\Xi$.

\section{III.C. EFIT Reconstruction}

Preliminary equilibria have been calculated for SSX using the EFIT equilibrium code, a Grad-Shafranov solver developed at General Atomics Technologies. ${ }^{16}$ EFIT may be used either to calculate a theoretical equilibrium or to fit an equilibrium to experimental measurements. The pressure $P$ and the current function $F F^{\prime}$ are expanded as polynomials in the normalized stream function:

$$
\chi=\frac{\psi_{\text {mag }}-\psi}{\psi_{\text {mag }}-\psi_{\text {sep }}},
$$

where $\psi_{\text {mag }}$ is $\psi$ evaluated at the magnetic axis and $\psi_{s e p}$ is $\psi$ evaluated at the separatrix.

EFIT has been modified by Zhang for use on the magnetized injector of the Helicity Injected Tokamak experiment. These modifications have been briefly described ${ }^{17}$ and are the subject of a paper in preparation. ${ }^{18}$ In short, they involve separately parameterizing the current function $F F^{\prime}$ for the gun and confinement regions. For the gun region, defined by $\psi>\psi_{\text {sep }}$ and $z<z_{\text {sep }}$, where $z_{\text {sep }}$ 
is the extreme axial location of the separatrix, one polynomial is used, while another is used for the confinement region. These modifications also render EFIT suitable for modeling the magnetized gun characteristic of CTX-type spheromaks.

Although designed to compute equilibria for tokamaks, it can be readily adapted for use on spheromaks. ${ }^{19}$ To do this, the magnitude of the externally applied toroidal $B$ field is set to some small value $(\sim 1 \mathrm{mG}$ on the magnetic axis), and a small cylindrical limiter (4 $\mathrm{mm}$ in diameter) is included along the axis of the device to exclude the region where the toroidal field diverges. For

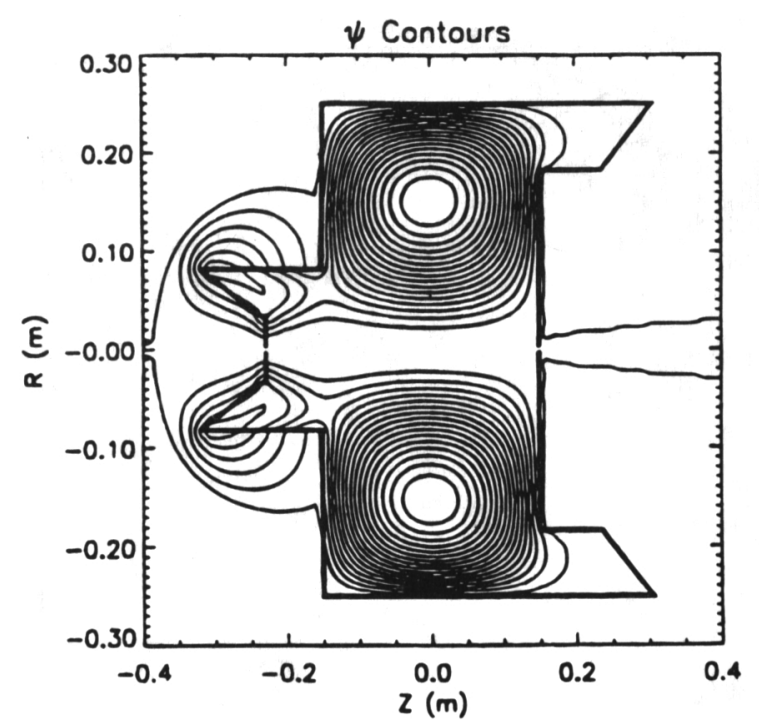

(a)

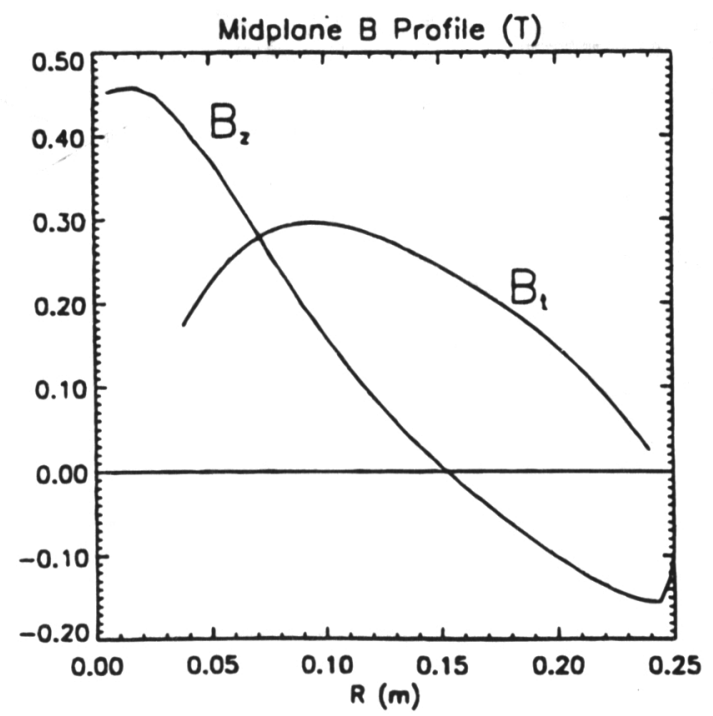

(c) this calculation, the formation and sustainment guns are treated separately, and the pressure is neglected. For both equilibria, the proper parameterizations for $F F^{\prime}$ were found by trial and error. The flux conserver has a radius $R$ of $0.25 \mathrm{~m}$ and a height $L$ of $0.30 \mathrm{~m}$; it therefore meets the tilt-stability criterion: $L / R=1.2<1.67$.

The equilibrium driven by the formation gun (Fig. 1a) uses the following parameterization for $F F^{\prime}: F F^{\prime}=\beta_{0}$ in the formation gun, and $F F^{\prime}=\gamma_{0}+\gamma_{1} \chi-\left(\gamma_{0}+\gamma_{1}\right) \chi^{2}$ in the confinement region. The coefficients $\beta_{0}, \gamma_{0}$, and $\gamma_{1}$ are optimized by EFIT to yield a solution that best fits a set of constraints (in this case the toroidal plasma current, the

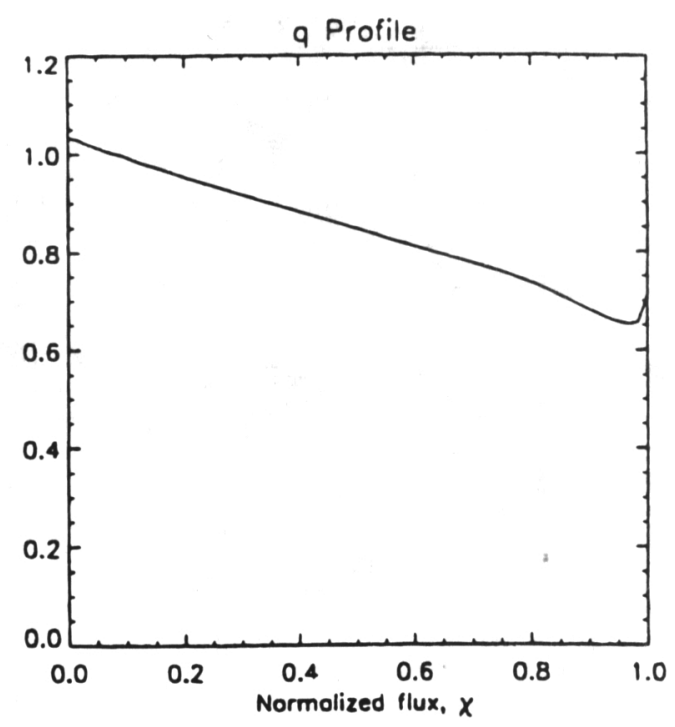

(b)

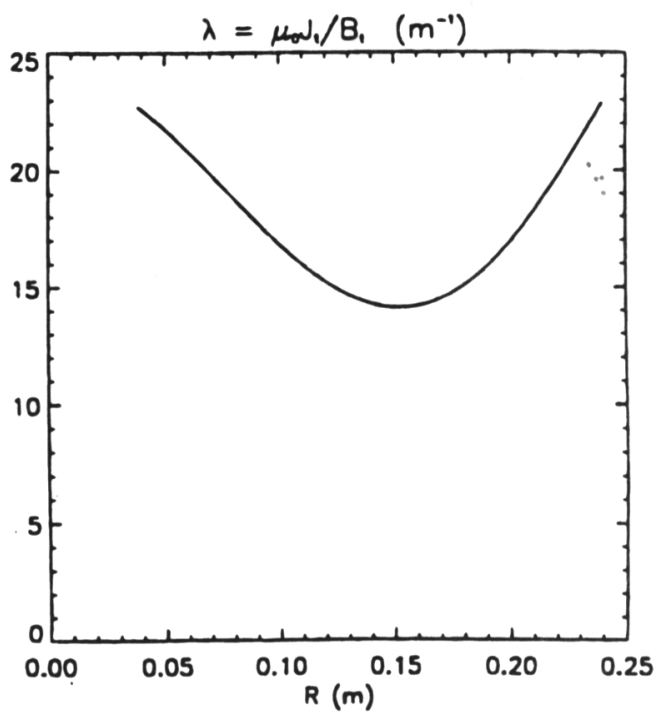

(d)

Fig. 1. Spheromak driven by formation gun alone: (a) $\psi$ contours (the limiter used in the calculation is shown in bold lines), (b) safety factor $q$ profile, (c) toroidal and poloidal B field profiles along midplane ( $B_{t}$ is shown only within the separatrix), and (d) $\lambda$ profile within separatrix. 
gun current, and $\psi$ at the wall). As EFIT was written for tokamaks, its standard form for $F F^{\prime}$ includes a constant term to account for the sizeable poloidal current of the toroidal field coil. The form of $F F^{\prime}$ we have chosen for the confinement region is such that $F F^{\prime}$ vanishes at the separatrix, which tends to suppress the constant term. The ultimate justification for the chosen $F F^{\prime}$ is that it yields $\lambda$ and $q$ profiles consistent with a driven spheromak. The formation gun has the following dimensions: $r_{\text {inner }}=0.0335 \mathrm{~m}$ and $r_{\text {outer }}=0.082 \mathrm{~m}$. The bias flux is $3 \mathrm{mWb}$, and the gun current is $99.8 \mathrm{kA}$, yielding $\lambda_{\text {gun }}=41.8 \mathrm{~m}^{-1}$. The spheromak equilibrium has a plasma current of $142 \mathrm{kA}$ and a closed poloidal flux of $10.5 \mathrm{mWb}$ with a diverted edge flux of 1.7 $\mathrm{mWb}, 57 \%$ of the bias flux. The object has $q$ and B-field profiles (Figs. lb and lc) typical of spheromaks and a hollow $\lambda$ profile (Fig. 1d). The average spheromak $\lambda$ is 17.1 $\mathrm{m}^{-1}$, yielding a geometric efficiency of

$$
\epsilon=\frac{\lambda_{\text {sph }}}{\lambda_{\text {gun }}}=\frac{17.1 \mathrm{~m}^{-1}}{41.8 \mathrm{~m}^{-1}}=41 \%
$$

The equilibrium driven by the sustainment gun (Fig. 2a) uses a different parameterization for $F F^{\prime}: F F^{\prime}=$ $\gamma_{0}+\gamma_{1} \chi-\left(\gamma_{0}+\gamma_{1}\right) \chi^{2}$ for both the gun and confinement

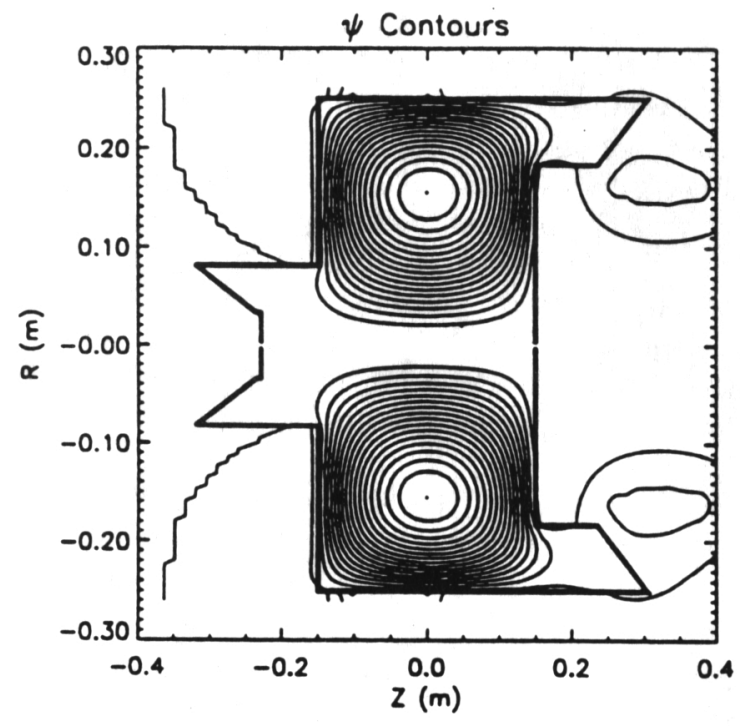

(a)

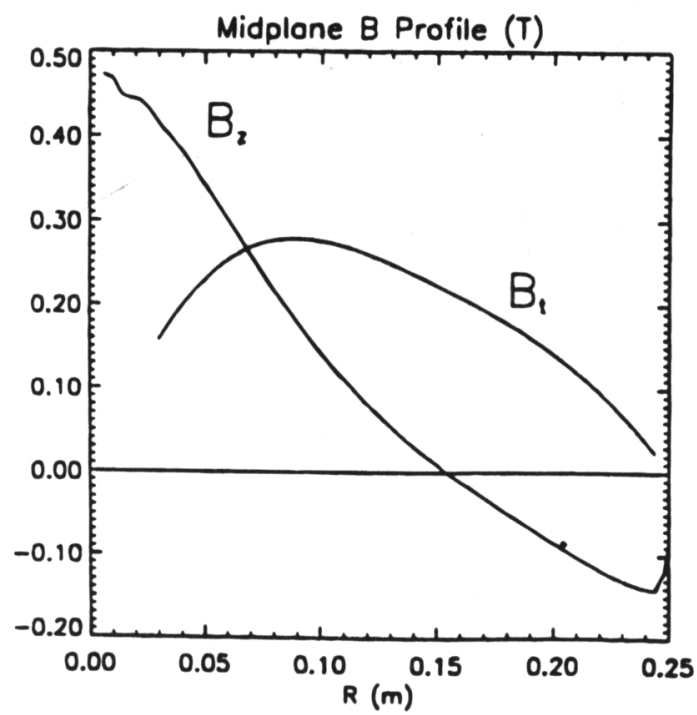

(c)

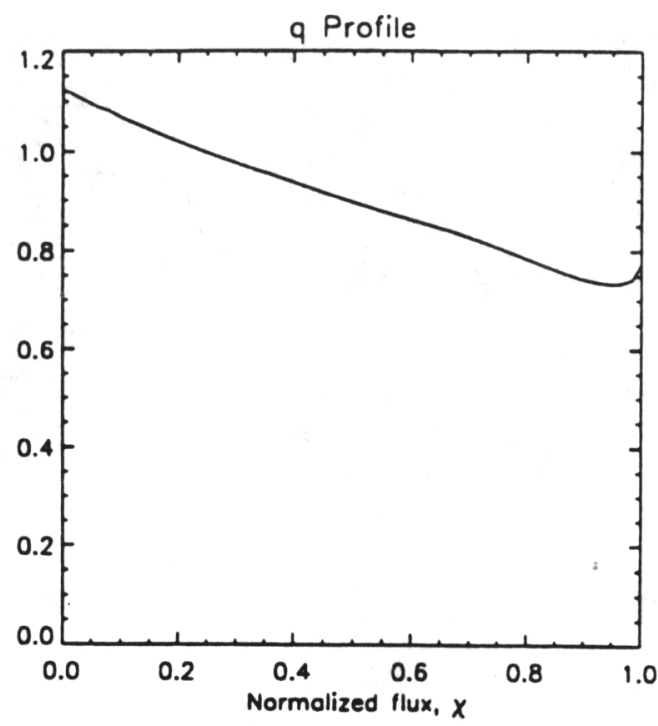

(b)

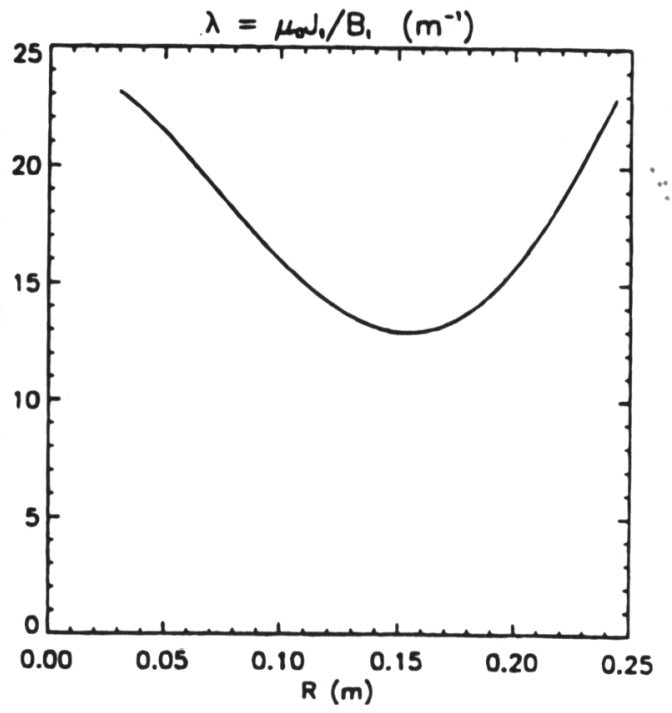

(d)

Fig. 2. Spheromak driven by sustainment gun alone: (a) $\psi$ contours (the limiter used in the calculation is shown in bold lines), (b) safety factor $q$ profile, (c) toroidal and poloidal B field profiles along midplane ( $B$, is shown only within the separatrix), and (d) $\lambda$ profile within separatrix. 
regions. The sustainment gun has dimensions as follows: $r_{\text {inner }}=0.183 \mathrm{~m}$ and $r_{\text {outer }}=0.25 \mathrm{~m}$. The bias flux is $1.05 \mathrm{mWb}$, and the gun current is $19.9 \mathrm{kA}$, yielding $\lambda_{\text {gun }}=23.8 \mathrm{~m}^{-1}$. The spheromak equilibrium has a plasma current of $143 \mathrm{kA}$ and a closed poloidal flux of $10.0 \mathrm{mWb}$. In this case, all of the bias flux is diverted around the spheromak. The plasma current and poloidal flux as well as the $q$, B-field, and $\lambda$ profiles (Figs. $2 \mathrm{~b}, 2 \mathrm{c}$, and $2 \mathrm{~d}$ ) are similar to those for the object driven by the formation gun. The average $\lambda$ for the spheromak is $16.4 \mathrm{~m}^{-1}$, yielding a geometric efficiency

$$
\epsilon=\frac{16.4 \mathrm{~m}^{-1}}{23.8 \mathrm{~m}^{-1}}=69 \% \text {. }
$$

Although chosen as a free parameter, the gun currents in each of the two equilibria are not arbitrary for the given geometry: No equilibrium could be found with the formation gun driven at lower current, nor could a solution be found with the sustainment gun driven at currents significantly higher than $20 \mathrm{kA}$. A sharp boundary model of the sustainment gun ${ }^{13}$ gives a threshold $\lambda$ of $45 \mathrm{~m}^{-1}$, corresponding to a gun current $I_{g u n}=41 \mathrm{kA}$. However, no equilibrium could be found with sustainment gun currents this high.

These results suggest that the larger sustainment gun indeed couples more efficiently to the spheromak and drives a given toroidal plasma current for a significantly lower gun current than does the smaller formation gun. Both guns in the simulation generate spheromaks with plasma current of $\sim 140 \mathrm{kA}$ and poloidal flux of $\sim 10 \mathrm{mWb}$, but the larger gun does it with significantly less power.

Recent theoretical work ${ }^{20}$ sheds new light on minimum energy states in driven plasmas and suggests that nonmonotonic $\lambda$ profiles are possible. These profiles ex- hibit greater magnetic shear and may have better confinement properties than constant or hollow $\lambda$ profiles. Internal magnetic measurements used with EFIT will be necessary to definitively measure the $\lambda$ profile. Further investigation of the $\lambda$ profile in driven plasmas would be of great value to fusion research generally.

\section{EXISTING FACILITY}

The present status of the Swarthmore Magnetofluids Laboratory and the SSX is reviewed here (see Fig. 3).

The versatile stainless steel vacuum chamber is $\sim 1 \mathrm{~m}$ long and $0.6 \mathrm{~m}$ in diameter. Two removable endplates afford access to a variety of flux conservers inside. The California Institute of Technology (Caltech) group ${ }^{21}$ and the CTX group ${ }^{7,8,10,11}$ have demonstrated the critical role of good vacuum technique, wall conditioning, and titanium gettering in spheromak research. We have decided to use cryo and sorption pumps in an effort to eliminate wall contamination due to pump oil. We also will be using a titanium-gettering system so that the inner wall of the flux conserver can be periodically coated with titanium.

The initial flux conserver will be a 0.16 -m-diam right circular copper cylinder (what the LANL group called the "tuna can"). The initial goal will be to form a spheromak, which is stable to the tilt, and map out the equilibrium structure with magnetic probes. For the spheromak to be stable to the tilt, the length-to-radius ratio should be $^{22} L / R<1.67$, so the length of the 0.16 -m-diam tuna can should be $\sim 0.12 \mathrm{~m}$. The second generation of flux conservers will be $0.5 \mathrm{~m}$ in diameter. These will be custom rolled at Swarthmore and coated with tungsten or tantalum.

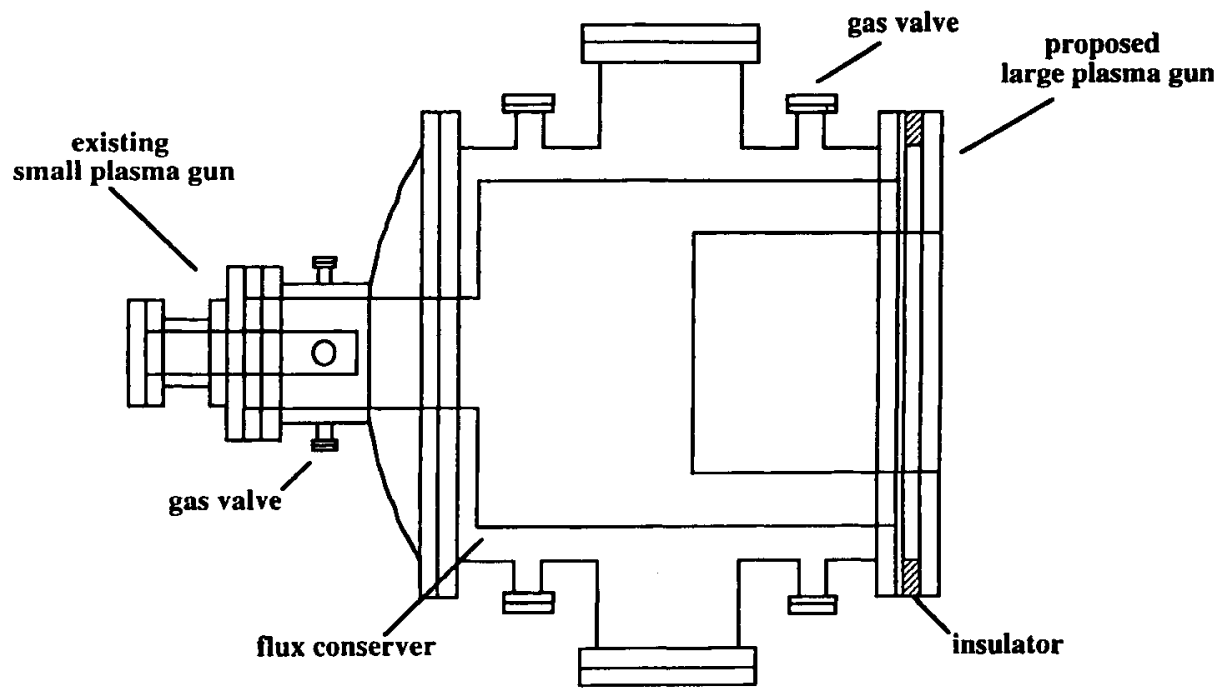

Fig. 3. Diagram of the SSX. The existing small magnetized plasma gun $(r=0.08 \mathrm{~m})$ is indicated on the left, and the large magnetized plasma gun $(r=0.25 \mathrm{~m})$ is indicated on the right. 
It is important to use gas valves that open quickly and have a high flow rate. At a density of a few $10^{21} \mathrm{~m}^{-3}$, our first spheromak magnetofluids will have a particle inventory of $\sim 10^{19}$ protons. This inventory corresponds to an initial neutral gas load of $\sim 1 \mathrm{~cm}^{3}$ at $1 \mathrm{~atm}$. The neutral gas will flow through the gun and into the flux conserver in $<500 \mu$ s (sound speed), so the valve needs to fully open in a time that is short compared with this $(\sim 100 \mu \mathrm{s})$. The valve also needs to maintain a high vacuum seal when not in use. Valves similar to those used on the Caltech CT injector and based on valves used on the Canadian CT injector ${ }^{23}$ are currently being designed to meet these criteria.

The gun magnetic flux or "stuffing flux" will be provided by an external coil and a cylinder of high magnetic permeability material (Permendur V) inside the center electrode. The coil will be energized by a small auxiliary capacitor bank fired by a silicon controlled rectifier (several millifarads at a few hundred volts). We need to supply up to several milliwebers of flux through the 0.064-m (2.5-in.)-diam inner electrode (up to $\sim 2 \mathrm{~T}$ magnetic field in the gun).

The inner and outer electrodes will be copper with a refractory coating. We have experimented with several electrode coatings ${ }^{21}$ and have had good results with chrome, tungsten, and tantalum. We require that the coating have a high melting point, low sputtering coefficient, and good thermal and electrical conductivity. Coating small electrodes with tungsten has proven effective and inexpensive. We will use high vacuum ceramic breaks to isolate the electrodes.

The most critical part of the experiment is the pulsed, high-voltage power supply. We need to apply up to $10 \mathrm{kV}$ between the inner and outer electrodes to break down the gas and create the plasma. We need to supply up to $100 \mathrm{kA}$ of current for up to $100 \mu \mathrm{s}$ (a few coulombs of charge), so we require several $100 \mu \mathrm{F}$ of capacitance. Because we will be injecting the spheromak directly into a copper flux conserver and stalling it there, we do not need a particularly fast power supply. Relaxing the constraint of short rise time (low inductance) makes the power supply easier to build and more reliable.

There are suitable power supplies available at LANL that were built for the FRX-C compression experiment ${ }^{24}$ there in the late $1980 \mathrm{~s}$. They feature a $10-\mathrm{kV}, 0.5-\mathrm{mF}$, 25-kJ capacitor switched and crowbarred by two large, water-cooled, $D$-size ignitrons. Each module is capable of supplying over $100 \mathrm{kA}$ of current in $50 \mu \mathrm{s}$.

The principal diagnostics needed for the proposed experiment are voltage probes and current monitors to characterize gun impedance and power. We will also characterize the spheromak equilibrium with magnetic probes and reconstruct the equilibrium with EFIT, which may be used to find the equilibrium that best fits a set of measurements. From these equilibria, the spheromak $\lambda$ may be determined and, from that, the geometric efficiency $\epsilon$. EFIT has so far proved satisfactory for use on spheromaks; it is flexible and is maintained on an ongoing basis by General Atomics Technologies. If it proves necessary to use an equilibrium code written expressly for spheromaks, we have one available written by Marklin for use on CTX.

A thorough understanding of spheromak physics requires careful measurements of the gun parameters. In particular, the Morozov or Hall parameter $\Xi$ has been identified as important in characterizing the gun operation; it depends on the mass flow rate of the injected plasma as well as the gun current. A method for measuring the average mass flow rate has been used on the CTX experiment at LANL (Ref. 25). It involves using a sensitive piezoelectric pressure transducer to measure the duration $\Delta t$ of the gas pulse into the gun. From this $\Delta t$ and the total gas inventory, an average flow rate is calculated, which is found to vary linearly with the fill pressure of the gas plenum. These measurements will be correlated with the number density, measured with a $\mathrm{He}-\mathrm{Ne}$ laser interferometer. Both a $0.63-\mu \mathrm{m}$ line and a $3.39-\mu \mathrm{m}$ line will be available.

The spheromak gun in this facility is similar to guns built at Caltech, so we have some confidence in how the guns will perform. A $10-\mathrm{kV}, 500-\mu \mathrm{F}$ pulsed-power supply should generate a spheromak with internal currents of up to $100 \mathrm{kA}$, internal fields of $0.2 \mathrm{~T}$, densities up to $10^{21} \mathrm{~m}^{-3}$, and with lifetimes up to $100 \mu \mathrm{s}$. The electron temperature will be in the range of 20 to $50 \mathrm{eV}$ with proper wall conditioning. The spheromak will break off of the gun in a few microseconds and move into the flux conserver at its Alfvén velocity $(\sim 0.03 \mathrm{~m} / \mu \mathrm{s}$ in this case). The expected parameters of the Swarthmore spheromak are summarized in Table I.

TABLE I

SSX Parameters

\begin{tabular}{|l|l|}
\hline \multicolumn{1}{|c|}{ Parameter } & Value (Two Guns) \\
\hline Density & $10^{20}$ to $10^{21} \mathrm{~m}^{-3}$ \\
Temperature & 20 to $50 \mathrm{eV}$ \\
B field (typical) & $0.2 \mathrm{~T}$ \\
$r_{g u n}$ & $0.08 \mathrm{~m} ; 0.25 \mathrm{~m}$ \\
$\lambda_{g u n}$ & $40 \mathrm{~m}^{-1} ; 12 \mathrm{~m}^{-1}$ \\
$I_{g u n}$ & $100 \mathrm{kA} ; 20 \mathrm{kA}$ \\
$\Phi_{g u n}$ & $3 \mathrm{mWb} ; 1 \mathrm{mWb}$ \\
$r_{s p h}$ & $0.25 \mathrm{~m}$ \\
$\lambda_{s p h}$ & $12 \mathrm{~m}{ }^{-1}$ \\
$I_{s p h}$ & $100 \mathrm{kA}$ \\
$\Phi_{s p h}$ & $10 \mathrm{mWb}$ \\
$\epsilon$ & $0.3 ; 1$ \\
$\Xi$ & 0.1 \\
$R_{m}$ & 200 to 2000 \\
Volume & 10 to $100 \ell$ \\
Particle inventory & $10^{19}$ to $10^{20}$ protons \\
Power modules (four) & $10 \mathrm{kV} ; 25 \mathrm{~kJ}$ \\
\hline
\end{tabular}




\section{PROPOSED EXPERIMENT}

The goal of the proposed experiment will be to form and sustain spheromaks with a variety of sources (spheromak guns) and compare the efficiencies, impedances, and $\lambda$ for each (as defined in Sec. III earlier). As research in sustained spheromaks is reestablished in the coming years, it will be important to verify and add to the existing database. In particular, we would like to provide the proof of principle of the idea of separate sources for formation and sustainment (see Fig. 3). We wish to demonstrate that a spheromak formed by the small gun can be sustained by the large gun with much less power.

The small formation gun will operate at high voltage and high $\Phi_{\text {gun }}$. The high voltage will ensure reliable breakdown of the gas. High voltage coupled with high flux should provide a rapid rate of helicity buildup in the spheromak since $\partial K / \partial t \cong 2 V_{g u n} \Phi_{g u n}$ initially (when $K_{s p h}$ is small). This formation process is analogous to current "rampup" in tokamaks. We expect, however, that the small gun will form the spheromak inefficiently because the size of the gun is much less than the size of the spheromak it is forming. We will use one of the $10-\mathrm{kV}, 25-\mathrm{kJ}$ modules for formation.

The larger sustainment gun will operate at low voltage and low $\Phi_{\text {gun }}$. We will use three of the power modules in parallel with additional capacitance (perhaps $5-\mathrm{mF}$ total) and operate at $<2 \mathrm{kV}$ ( $10 \mathrm{~kJ}$ of stored energy). The lower voltage allows us to use simpler insulators. The lower expected current density should cause less ablation of electrode material. In steady state, we expect that helicity injection from the large sustainment gun will simply balance the (small) rate of helicity dissipation in the spheromak: $2 V_{\text {gun }} \Phi_{\text {gun }} \cong K_{\text {sph }} / \tau_{K}$. This is analogous to steady-state current drive in tokamaks. We also expect that this process will be efficient so that gun power at the source is simply balanced by the (small) rate of energy dissipation in the spheromak: $I_{g u n} V_{g u n} \cong W_{s p h} / \tau_{K}$. The required gun power should be low since we expect $\tau_{K}$ for the warm, fully formed spheromak to be large. If $W_{s p h}$ is $1 \mathrm{~kJ}$ and $\tau_{K}$ is $100 \mu \mathrm{s}$, then the gun power is $10 \mathrm{MW}$. (perhaps $10 \mathrm{kA}$ at $1 \mathrm{kV}$ depending on the gun impedance). By comparison, the initial formation power might be as high as $1 \mathrm{GW}$ (perhaps $100 \mathrm{kA}$ at $10 \mathrm{kV}$ ).

Another advantage of matching $\lambda_{\text {gun }}$ and $\lambda_{s p h}$ is that magnetic turbulence (in the form of kink modes) is known ${ }^{26}$ to be driven by $\nabla \lambda$. This is clear since we normally think of kinks being driven by large gradients in the current density $J$ (which is closely related to $\lambda$ ). Indeed, the reason small guns are inefficient at sustaining large spheromaks is because they drive kinks. In the CTX spheromak, hollow $\lambda$ profiles were observed during sustainment (more current density on the edge, $n=1$ modes), and peaked $\lambda$ profiles were observed during decay (less current density on the edge, $n=2$ modes). In both cases, MHD modes were driven unstable as the system tried to relax back to a flat $J / B$ profile consistent with the Taylor state. It is for this reason that we would like $\lambda_{g u n}$ and $\lambda_{s p h}$ to be matched and the gun and spheromak to be the same size.

We should point out that there are pathologies if $\epsilon \rightarrow 1$ identically. The approach to the force-free state is driven by turbulence, and the free energy for the turbulence comes from the mismatch in $\lambda$. Another way to say this is that the spheromak moves "downhill" in $\lambda$, and magnetic energy is dissipated as the spheromak relaxes. If the gun and spheromak had identical $\lambda$ 's, then the geometric efficiency $\epsilon$ would be identically unity, but there would be no free energy to drive turbulent relaxation. In this case, helicity would not flow from the gun to the spheromak.

Once we have demonstrated that we can form spheromaks in simple flux conservers, we will modify the large flux conserver to accommodate a large gun. The large gun will be constructed with relatively modest requirements for voltage insulation and gun flux. It will require a flux-conserving jacket (copper) and a thin coating of a refractory metal (tungsten). The current density on the large gun electrodes will be relatively small, so we may be able to operate with a simple coating such as chrome or rhodium. ${ }^{21}$

We propose to form a spheromak in the usual way with a small gun while monitoring $I_{\text {gun }}$ and $V_{\text {gun }}$. The equilibrium will be monitored with a few magnetic probes near the wall of the flux conserver and reconstructed with EFIT, and the threshold value of $\lambda_{\text {gun }}$ will be determined. Once equilibrium is established, the small gun will be crowbarred, and the large, low-voltage gun will be energized. Again, $I_{\text {gun }}$ and $V_{\text {gun }}$ will be monitored, and any adverse effects on equilibrium will be measured with the magnetic probes and EFIT. It will be important to calculate the gun power, impedance, and $\lambda$ from these measurements. In particular, it will be interesting to compare the threshold value of $\lambda_{\text {gun }}$ for the large and small guns.

As discussed in Sec. III, we expect $V_{\text {gun }}$ to have a strong nonlinear dependence on $I_{\text {gun }}$. We also expect $V_{g u n}$ to depend on the mass flow rate $\dot{m}$. While it is difficult to measure $\dot{m}$ directly, it will be important to measure the line-averaged electron density $n_{e}$ as a function of time and correlate it with the mass flow rate.

It has been suggested that although the global confinement time of spheromaks is not attractive for a fusion reactor, it is possible that core confinement is very good. ${ }^{27}$ The fact that ${ }^{11} T_{e} \sim 400 \mathrm{eV}$ and that hard $\mathrm{X}$ rays ${ }^{28}$ were observed in decaying CTX spheromaks suggests good core confinement during decay. However, this observation raises the following question: Why was good confinement not observed during sustainment? It is possible that poor $\lambda$ matching caused turbulence that degraded confinement, but this is still an open question. It is clear that well-diagnosed, sustained spheromak experiments are an important next step for the alternate concept fusion effort.

We are at a stage in spheromak research in the 1990s analogous to the stage of tokamak research in the 1970s. 
In the 1970 s, tokamak researchers were able to generate hot tokamak plasmas but were yet to discover steadystate current drive techniques. In the 1990s, spheromak researchers are able to generate hot spheromak plasmas but not in a sustained discharge. It is important to understand spheromak sustainment if spheromaks are to become a viable alternative in magnetic confinement fusion.

\section{CONCLUSION}

A spheromak experiment has been described that incorporates separate magnetized plasma guns for formation and sustainment. Once formed with a small gun, we expect that the spheromak can be sustained against dissipation with a larger gun using much less power. We expect that the spheromak equilibrium will be sustained by the large gun without adverse effects (due to either impurity influx from the walls or poor $\lambda$ matching to the flux conserver).

\section{ACKNOWLEDGMENTS}

This work was supported by awards from the U.S. Department of Energy, Research Corporation, and the Petroleum Research Fund. The authors wish to thank C. W. Barnes, T. R. Jarboe, and B. A. Nelson for useful discussions and C. Zhang and L. Lao for help with EFIT. The technical assistance of S. Palmer for the construction of flux conservers and electrodes is gratefully acknowledged.

\section{REFERENCES}

1. J. B. TAYLOR, "Relaxation and Magnetic Reconnection in Plasmas," Rev. Mod. Phys., 58, 741 (1986).

2. J. B. TAYLOR, "Relaxation of Toroidal Plasma and Generation of Reverse Magnetic Fields," Phys. Rev. Lett., 33, 1139 (1974).

3. T. R. JARBOE et al., "Motion of a Compact Toroid Inside a Cylindrical Flux Conserver," Phys. Rev. Lett., 45, 1264 (1980).

4. M. YAMADA et al., "Quasistatic Formation of the Spheromak Plasma Configuration," Phys. Rev. Lett., 46, 188 (1981).

5. T. R. JARBOE, "Review of Spheromak Research," Plasma Phys. Control. Fusion, 36, 945 (1994).

6. T. R. JARBOE et al., "The Ohmic Heating of a Spheromak to 100 eV," Phys. Fluids, 27, 13 (1984).

7. C. W. BARNES et al., "Spheromak Formation and Operation with Background Filling Gas and a Solid Flux Conserver in CTX," Nucl. Fusion, 24, 267 (1984).

8. C. W. BARNES et al., "Zero-Dimensional Energy Balance Modelling of the CTX Spheromak Experiment," Nucl. Fusion, 25, 1657 (1985).
9. F. J. WYSOCKI et al., "Improved Energy Confinement in Spheromaks with Reduced Field Errors," Phys. Rev. Lett., 65, 40 (1990).

10. F. J. WYSOCKI et al., "Evidence for a Pressure-Driven Instability in the CTX Spheromak," Phys. Rev. Lett., 61, 2457 (1988).

11. T. R. JARBOE et al., "Progress with Energy Confinement Time in the CTX Spheromak," Phys. Fluids, B2, 1342 (1990).

12. T. R. JARBOE et al., "Slow Formation and Sustainment of Spheromaks by a Coaxial Magnetized Plasma Source," Phys. Rev. Lett., 51, 39 (1983).

13. C. W. BARNES et al., "The Impedance and Energy Efficiency of a Coaxial Magnetized Plasma Source Used for Spheromak Formation and Sustainment," Phys. Fluids, B2, 1871 (1990).

14. C. W. BARNES et al., "Experimental Determination of the Conservation of Magnetic Helicity from the Balance Between Source and Spheromak," Phys. Fluids, 29, 3415 (1986).

15. M. R. BROWN and P. M. BELLAN, "Efficiency and Scaling of Current Drive and Refueling by Spheromak Injection into a Tokamak," Nucl. Fusion, 32, 1125 (1992).

16. L. L. LAO et al., "Reconstruction of Current Profile Parameters and Plasma Shapes in Tokamaks," Nucl. Fusion, 25, 11 (1985).

17. C. ZHANG, T. R. JARBOE, and B. A. NELSON, "Equilibrium Studies on the Helicity Injected Tokamak," Bull. Am. Phys. Soc., 38, 2010 (1993).

18. C. ZHANG, A. MARTIN, T. R. JARBOE, B. A. NELSON, and D. J. ORVIS, "Equilibrium Studies on the Helicity Injected Tokamak" (in preparation).

19. L. L. LAO, General Atomics Technologies, Personal Communication (Mar. 1995).

20. P. K. BROWNING, P. A. FRENCH, and T. R. JARBOE, "Minimum Energy States in Spheromaks with External Driving," Proc. 20th Europhysics Conf. Controlled Fusion and Plasma Physics, Vol. II, p. 503.

21. M. R. BROWN, A. D. BAILEY III, and P. M. BELLAN, "Characterization of a Spheromak Plasma Gun: The Effect of Refractory Electrode Coatings," J. Appl. Phys., 69, 6302 (1991).

22. J. M. FINN et al., "Spheromak Tilting Instability in Cylindrical Geometry," Phys. Fluids, 24, 1336 (1981); see also A. BONDESON et al., "Tilting Instability of a Cylindrical Spheromak," Phys. Fluids, 24, 1682 (1981).

23. J. C. THOMAS et al., "A Simple Fast Pulse Gas Valve Using Dynamic Pressure Differential as the Primary Closing Mechanism," Rev. Sci. Instrum., 64, 1410 (1993).

24. D. J. REJ et al., "Design and Performance of the $10 \mathrm{kV}$, 5 MA Pulsed Power System for the FRX-C Compression Experiment," LA-1 1519-MS, Los Alamos National Laboratory (1989). 
25. R. HOYT, "Optimization of Applied Magnetic Nozzles for Coaxial Plasma Accelerators," PhD Thesis, University of Washington (1994).

26. S. O. KNOX et al., "Observations of Spheromak Equilibria Which Differ from the Minimum-Energy State and Have Internal Kink Distortions," Phys. Rev. Lett., 56, 842 (1986).
27. E. B. HOOPER, J. H. HAMMER, C. W. BARNES, J. C. FERNÁNDEZ, and F. J. WYSOCKI, "A Reexamination of Spheromak Experiments and Opportunities," Fusion Technol., 29, 191 (1996).

28. R. E. CHRIEN et al., "Evidence for Runaway Electrons in a Spheromak Plasma," Nucl. Fusion, 31, 1390 (1991).

Michael R. Brown (PhD, Dartmouth College, 1987) is an assistant professor of physics at Swarthmore College. His interests include the basic physics of reconnection and relaxation in magnetofluids and the study of alternate concepts for magnetic confinement fusion.

Adam K. Martin is a graduate student in physics at the University of Washington. His interests include alternate devices for magnetic confinement fusion and plasma propulsion. 